\title{
Pulmonary complications in patients with haematological malignancies treated at a respiratory ICU
}

\author{
S. Ewig*, A. Torres*, R. Riquelme*, M. El-Ebiary*, M. Rovira**, E. Carreras**, \\ A. Raño*, A. Xaubet*
}

Pulmonary complications in patients with haematological malignancies treated at a respiratory ICU. S. Ewig, A. Torres, R. Riquelme, M. El-Ebiary, M. Rovira, E. Carreras, A. Raño, A. Xaubet. OERS Journals Ltd 1998.

ABSTRACT: Patients with haematological malignancies developing severe pulmonary complications have a poor outcome, especially after bone-marrow transplantation (BMT). We studied the aetiology, the yield of different diagnostic tools, as well as the outcome and prognostic factors in the corresponding population admitted to our respiratory intensive care unit (RICU).

Overall, 89 patients with haematological malignancies and pulmonary complications treated within a $10 \mathrm{yr}$ period were included.

The underlying malignancies were predominantly acute leukaemia and chronic myeloid leukaemia $(66 / 89,74 \%)$. Fifty-two of $89(58 \%)$ patients were bone marrow recipients. An aetiological diagnosis could be obtained in 61/89 (69\%) of cases. The aetiology was infectious in 37/89 (42\%) and noninfectious in $24 / 89(27 \%)$. Blood cultures and cytological examinations of bronchoalveolar lavage fluid were the diagnostic tools with the highest yield $(13 / 43(30 \%)$ and 13/45 (29\%) positive results, respectively). Necropsy results were coincident with results obtained during the lifetime in $43 \%$ of cases with infectious and $60 \%$ with noninfectious aetiologies. Overall mortality was $70 / 89(79 \%)$, and $47 / 52(90 \%)$ in transplant recipients. The requirement of mechanical ventilation, BMT, and an interval $<90$ days of BMT prior to ICU admission were independent adverse prognostic factors.

The outcome in this patient population was uniformly poor. It was worst in bone marrow recipients developing pulmonary complications $<\mathbf{9 0}$ days after transplantation and requiring mechanical ventilation. Decisions about intensive care unit admission and mech-anical ventilation should seriously consider the dismal prognosis of these patients.

Eur Respir J 1998; 12: 116-122.

The treatment of haematological malignancies is frequently associated with pulmonary complications, including infectious and noninfectious aetiologies [1-4]. The prognosis of these patients worsens significantly when respiratory failure requiring intensive care unit (ICU) treatment and mechanical ventilation occurs $[5,6]$. Despite adequate diagnostic evaluation and treatment in an ICU, mortality remains so high that the benefits of the use of mechanical ventilation have been seriously questioned. This is especially true for bone-marrow transplant recipients [7-11].

We, therefore, report our experience in the treatment of patients with haematologic malignancies and pulmonary complications admitted to our respiratory intensive care unit (RICU). We studied the aetiology of pulmonary compromise and determined the value of different diagnostic tools as well as the information obtained by necropsy. Moreover, we looked for prognostic factors associated with mortality.

\section{Materials and methods}

\section{Patient population}

The clinical charts of all patients with haematological malignancies and pulmonary complications admitted to our
*Servei de Pneumologia i Al.lergia Respiratoria and **Servei de Hematologia Clinica, Barcelona, Spain.

Correspondence: A. Torres

Hospital Clinic i Provincial

Servei de Pneumologia i Al.lergia Respiratoria Villaroel 170

08036 Barcelona

Spain

Fax: 3432275454

Keywords: Bone marrow transplantation haematological malignancy pneumonia

prognosis

Received: December 291997

Accepted after revision April 171998

Supported by IDIBAPS, Hospital Clinic, Barcelona, and CIRIT, Fundació Clinic.
ICU between January 1, 1984 and December 31, 1993 were retrospectively recorded. Overall, 89 patients were considered adequate for evaluation.

\section{Data collection}

In all cases, the following variables were recorded: age, sex, underlying haematological malignancy, presence or absence of bone marrow transplantation (BMT), allogeneic or autologous BMT and interval from BMT to ICU admission. From the variables available at admission, leucocyte count, platelet count, prothrombin, creatinine, arterial oxygen tension $\left(\mathrm{Pa}_{\mathrm{a}} \mathrm{O}_{2}\right) /$ inspiratory oxygen fraction $\left(F \mathrm{I}, \mathrm{O}_{2}\right)$ and the type (alveolar, interstitial, mixed), as well as, the distribution of radiographical infiltrates (uni- versus bilateral) were recorded. Chemotherapy and/or radiotherapy received, antimicrobial treatment during hospitalization, the absence or presence of adult respiratory distress syndrome (ARDS; as defined by MuRRAY et al. [12]), the requirement of mechanical ventilation, and the duration of mechanical ventilation were retrieved.

The aetiology of pulmonary compromise was classified as definitely infectious, noninfectious or undetermined. An aetiology was considered definite in the case of a 
microbiological confirmation of infection by respiratory or nonrespiratory samples and/or necropsy (see Diagnostic criteria). The diagnosis of a noninfectious aetiology was based on 1) the absence of a definite infectious aetiology; 2) evidence for a noninfectious aetiology as determined by clinical criteria and a compatible clinical course; or 3) necropsy results. Episodes that did not meet criteria for infectious or noninfectious aetiologies were classified as undetermined.

The clinical outcome was evaluated in terms of death in the ICU or discharge from ICU. Patients were grouped as survivors versus nonsurvivors, accordingly.

\section{Microbiological evaluation}

Noninvasive as well as invasive bronchoscopic evaluation was performed according to the clinical judgment of the physicians in charge.

Microbiological testing was performed as previously described [13]. In short, the respiratory samples were cultured for aerobic and anaerobic bacterial pathogens, mycobacteria, and fungi. In addition, they were evaluated by direct fluorescent antibody (DFA) technique for Legionella spp. Undiluted and serially diluted secretions were plated on blood-sheep agar, Wilkens-Chalgren, chocolate agar as well as Sabouraud's agar. All cultures were incubated at $37^{\circ} \mathrm{C}$ in aerobic and anaerobic culture and in a $\mathrm{CO}_{2}$-enriched atmosphere. Negative bacterial cultures were discarded after 5 days, and negative fungal cultures after 4 weeks.

Smears obtained by cytocentrifuge preparation were stained with Papanicolau, May-Grünwald Giemsa, PAS, haematoxylin-eosin, Perls, and Gomori-Grocott, and examined for cytological evidence of viral inclusion bodies, fungal, and parasitic infections as well as for the detection of siderophages and malignant cells.

\section{Diagnostic criteria}

Potentially pathogenic bacteria yielding Š $10^{5}$ colonyforming units (cfu) $\cdot \mathrm{mL}^{-1}$ in cultures of tracheobronchial aspirates (TBAS), $\breve{\mathrm{S}} 10^{3} \mathrm{cfu} \cdot \mathrm{mL}^{-1}$ in cultures of the protected specimen brush (PSB) as well as Š $10^{4} \mathrm{cfu} \cdot \mathrm{mL}^{-1}$ in cultures of bronchoalveolar lavage fluid (BALF) were considered as aetiological agents of pulmonary infiltrates. Any growth of facultative pathogenic bacteria such as Streptococcus viridans and Staphylococcus epidermidis was not considered as relevant. The identification of Legionella spp. and mycobacteria was accepted as diagnostic regardless of colony counts. The isolation of Candida spp. and Aspergillus spp. was considered as diagnostic in the presence of a compatible clinical and radiographical pattern. Pneumonia due to cytomegalovirus (CMV) was diagnosed in case of the demonstration of viral inclusion bodies in cytocentrifuge smears. Pneumocystis carinii pneumonia was diagnosed by a positive Gomori-Grocott stain. Pulmonary haemorrhage was detected by an excess of haemosiderin-laden macrophages recovered from BALF [14]. Any growth of bacteria or fungi in blood cultures (ex-cept S. epidermidis in only one blood culture) in the absence of another infectious focus was considered as presumptive aetiology of pulmonary complications (not necessarily pneumonia). Finally, pneumonia due to Candida spp., Aspergillus sp., CMV, and P. carinii was also established by its demonstration in post mortem lung histology.

\section{Prognostic analysis}

In the whole population, the parameters: mechanical ventilation, presence of BMT, and infectious versus noninfectious and undetermined aetiology were tested for an association with death. In both, patients without BMT and in bone marrow recipients, the following variables were tested: age, $\mathrm{Pa}, \mathrm{O}_{2} / F \mathrm{I}, \mathrm{O}_{2}$ at admission, leucocyte and platelet count as well as prothrombin time and creatinine at admission, uni- versus bilateral infiltrates in chest radiographs, bacteraemia and fungaemia, infectious versus noninfectious and undetermined aetiology, and requirement of mechanical ventilation. In bone-marrow recipients, the parameters: allogeneic versus autologous transplantation, interval from BMT to ICU admission and history of graft-versus-host disease (GVHD) were tested additionally.

\section{Statistical analysis}

Nonparametric means were compared using Student's t-test. Categorical variables were compared using the chi-square test or Fisher's exact test where appropriate. Parameters significantly associated with death in univariate analysis were included in a multivariate analysis using a stepwise forward logistic regression. An $\alpha$-error of $<0.5$ was considered significant in all cases. All p-values reported are two-tailed.

\section{Results}

\section{General characteristics of the study population}

The underlying malignancies of the 89 patients (52 male, 37 female, mean age $36 \pm 15$ yrs, range $8-76$ yrs) were predominantly acute myeloid leukaemia (AML), acute lymphoblastic leukaemia (ALL), and chronic myeloid leukaemia (CML) (table 1). The majority of patients $(78 / 89,88 \%)$ had received chemotherapy with or without radiotherapy recently during the hospital stay. Fifty-two patients (58\%) had undergone BMT (43 allogeneic, nine autologous). The conditioning regimen was cyclophosphamide $120 \mathrm{mg} \cdot \mathrm{kg}^{-1}$ plus total body irradiation (TBI) with 12 Gy for allogeneic BMT and autologous BMT of acute leukaemia throughout the study period; patients with autologous BMT for lymphoma received only chemotherapy ("BEAC": 1,3-bis-(2chloroethyl)-1-nitrosourea (BCNU), etoposide, cytarabine, cyclophosphamide). Granulocyte colony stimulating factor (G-CSF) was regularly added since 1991 in patients with autologous BMT from day 7 until a documented neutrophil count $>1 \times 10^{9} \cdot \mathrm{L}^{-1}$ for three days. Twenty-five BMT

Table 1. - Underlying malignancies in 89 patients

\begin{tabular}{lrr}
\hline Underlying malignancy & $\mathrm{n}$ & $\%$ \\
\hline Acute myeloid leukaemia & 28 & 31.5 \\
Acute lymphoblastic leukaemia & 22 & 24.7 \\
Chronic myeloid leukaemia & 17 & 19.1 \\
Chronic lymphoid leukaemia & 3 & 3.4 \\
Hodgkin's lymphoma & 8 & 8.9 \\
Non-Hodgkin's lymphoma & 5 & 5.6 \\
Multiple myeloma & 3 & 3.5 \\
Aplastic anaemia & 2 & 2.2 \\
Myelodysplastic syndrome & 1 & 1.1 \\
Total & 89 & 100 \\
\hline
\end{tabular}


recipients (49\%) had already experienced GVHD. Bonemarrow recipients were significantly younger than patients without BMT $(29 \pm 11$ versus $46 \pm 15$ yrs, $\mathrm{p}<0.0001)$.

Patients were admitted to the ICU because of acute respiratory failure $(n=61$, including $n=23$ with ARDS), sepsis syndrome with and without septic shock $(n=11)$, pulmonary haemorrhage $(n=8)$, heart failure $(n=7)$, and miscellaneous conditions (fat embolism during bone marrow infusion and iatrogenic pneumothorax, $\mathrm{n}=1$ each). The mean $\mathrm{Pa}_{2} \mathrm{O}_{2} / \mathrm{FI}, \mathrm{O}_{2}$ at $\mathrm{RICU}$ admission was $21.7 \pm 9.6 \mathrm{kPa}(163 \pm 72$ $\mathrm{mmHg})$. Seventy-six patients $(85 \%)$ required mechanical ventilation.

At admission to the ICU, 73 patients (82\%) had a leucocyte count $<1 \times 10^{9}$ cells. $\mathrm{L}^{-1}$. All patients except one had abnormalities in chest radiographs. These were unilateral in $14(16 \%)$ and bilateral in $74(84 \%)$ of cases, and disclosed an alveolar consolidation in 57 cases $(65 \%)$, an interstitial pattern in $13(15 \%)$, and a mixed pattern in 18 (20\%).

The initial empirical antimicrobial treatment was standardized according to the following principles. Antibacterial treatment consisted of a combination regimen including a third-generation cephalosporin plus an aminoglycoside (preferably amikacin). Alternatives were an ureidopenicillin and ciprofloxacin. If Staphylococcus aureus was suspected or proven, vancomycin was added. In the case of nonresponse, antifungal treatment with amphothericin B was added empirically after the 4th or 5th day. Ganciclovir plus high-dose immunoglobulin (Ig)G-immunoglobulins were added since 1990 (and later on, foscarnet as an alternative) in the case of a suspected or proven CMV pneumonia. G-CSF was sporadically administered in patients with evidence of infection and prolonged neutropenia.

\section{Diagnostic procedures and number of necropsies}

Blood cultures for bacteria and fungi were obtained in 43 and TBAS in 34 patients. Flexible bronchoscopy was performed in 50 patients. The investigations included cultures of bronchoalveolar lavage fluid BALF in 49 cases, and cytological examinations of BALF in 44 cases. PSB was performed in 43 cases.

Overall, microbiological evaluation, including blood cultures and/or BALF, PSB and TBAS, was performed in 64 $(72 \%)$ patients. One patient had pulmonary tuberculosis. Thirty-one patients of 70 nonsurvivors (44\%) underwent necropsy.

\section{Aetiology of pulmonary infiltrates}

A definite aetiologic diagnosis of acute respiratory failure could be obtained by clinical, microbial as well as post mortem evaluation in 61/89 (69\%) of cases. It was infectious in 37 cases $(42 \%)$. Twenty-four definite noninfectious aetiologies $(27 \%)$ included diffuse alveolar damage (DAD) $(n=6)$, pulmonary haemorrhage $(n=8$, including two cases associated with DAD and bronchopneumonia, respectively), congestive heart failure $(n=7)$, and organizing pneumonia as well as severe fat embolism and iatrogenic pneumothorax in one case each. Twenty eight cases $(31 \%)$ remained undetermined, including 15 cases initially presenting with acute respiratory failure, five cases with ARDS and eight cases with sepsis.
Bacterial pneumonia was the most frequent aetiology $(\mathrm{n}=15$ plus six cases with mixed bacterial/opportunistic infections), followed by viral pneumonia ( $n=8$ plus three mixed infections), fungal pneumonia ( $n=7$ plus four mixed infections) and $P$. carinii pneumonia ( $\mathrm{n}=1$ plus one mixed infection). However, as regards pathogens, CMV was most frequently encountered $(\mathrm{n}=11)$, followed by Pseudomonas aeruginosa $(\mathrm{n}=10)$, and fungal pathogens (Candida spp. $\mathrm{n}=6$, Aspergillus spp. $\mathrm{n}=5)$. Ten of $37(27 \%)$ episodes accounted for mixed infections. The number of infectious aetiologies and pathogens is listed in table 2. Comparing patients with and without BMT, the only significant difference was a higher incidence of CMV in BMT patients $(\mathrm{p}=$ $0.007)$.

\section{Diagnostic yield of different techniques}

During lifetime, infectious aetiologies were identified in 28 patients. One single additional patient had known pulmonary tuberculosis. The infectious aetiology was established by blood cultures and TBAS as noninvasive diagnostic tools in 10/28 (36\%) cases and by bronchoscopy with PSB and/or BALF as invasive tools in 14/28 (50\%). In the remaining four cases $(14 \%)$, both procedures contributed to the diagnosis.

As regards to micro-organisms, blood cultures revealed a positive result in 13/43 (30\%). BALF yielded a positive result in $15 / 49(31 \%)$. Of these, culture results were positive in $2 / 49(4 \%)$ cases and cytological examinations in $13 / 45(29 \%)$ cases. PSB was culture-positive in 8/40 (20\%) cases and TBAS were culture-positive in 2/34 (6\%) cases.

Table 2. - Infectious aetiologies in 37 patients diagnosed during lifetime and at necropsy

\begin{tabular}{|c|c|c|}
\hline Pathogen & $\begin{array}{l}\text { Number } \\
\text { of cases }\end{array}$ & $\begin{array}{c}\text { Number of } \\
\text { pathogens }\end{array}$ \\
\hline Bacterial agents & 11 & 25 \\
\hline Streptococcus pneumoniae & 3 & 3 \\
\hline Streptococcus sanguis & 1 & 1 \\
\hline Streptococcus mitis & 1 & 1 \\
\hline Streptococcus viridans & 1 & 1 \\
\hline Enterococcus faecalis & & 3 \\
\hline Staphylococcus aureus & & 2 \\
\hline Serratia spp. & 1 & 1 \\
\hline Gram-negative enteric bacilli & 1 & 1 \\
\hline Pseudomonas aeruginosa & 2 & 10 \\
\hline Legionella pneumophilia & & 1 \\
\hline Mycobacterium tuberculosis & 1 & 1 \\
\hline Viral agents & 8 & 12 \\
\hline Cytomegalovirus (CMV) & 8 & 11 \\
\hline Herpes simplex virus & & 1 \\
\hline Fungal agents & 7 & 11 \\
\hline Candida spp. & 4 & 6 \\
\hline Aspergillus spp. & 3 & 5 \\
\hline Pneumocystis carinii & 1 & 2 \\
\hline Mixed infections & 10 & \\
\hline L. pneumophilia + E. faecalis & 1 & \\
\hline$S$. aureus $+P$. aeruginosa & 1 & \\
\hline E. spp. $+P$. aeruginosa & 2 & \\
\hline$S$. aureus $+P$. carinii & 1 & \\
\hline$P$. aeruginosa $+\mathrm{CMV}$ & 1 & \\
\hline$P$. aeruginosa + candida $\mathrm{spp}$ & 2 & \\
\hline P. aeruginosa + CMV + Aspergillus spp. & 1 & \\
\hline $\begin{array}{l}\text { P. aeruginosa }+\mathrm{CMV}+\text { Herpes } \\
\text { simplex virus }+ \text { Aspergillus spp. }\end{array}$ & 1 & \\
\hline
\end{tabular}


Table 3. - Pathogens retrieved by diagnostic techniques during lifetime

\begin{tabular}{lccccc}
\hline \multirow{2}{*}{ Pathogens } & BC & PSB & BAL & BAL & TBAS \\
& \multicolumn{5}{c}{ culture cytology } \\
& $\mathrm{n}=43$ & $\mathrm{n}=40$ & $\mathrm{n}=49$ & $\mathrm{n}=45$ & $\mathrm{n}=34$ \\
Streptococcus pneumoniae & 1 & 2 & - & - & - \\
Streptococcus sanguis & 1 & - & - & - & - \\
Streptococcus mitis & 1 & - & - & - & - \\
Streptococcus viridans & 1 & - & - & - & - \\
Enterococcus faecalis & 1 & - & - & - & - \\
Staphylococcus aureus & 1 & 1 & - & - & - \\
Serratia spp. & 1 & - & - & - & - \\
Pseudomonas aeruginosa & 3 & 4 & 1 & - & 1 \\
Gram-negative enteric & 1 & - & - & - & - \\
$\quad$ bacilli & & & & & \\
Legionella pneumophilia & - & - & - & - & 1 \\
Cytomegalovirus & - & - & - & 7 & - \\
Herpes simplex virus & - & - & - & - & - \\
Candida spp. & 2 & 1 & 1 & 3 & - \\
Aspergillus spp. & - & - & - & 1 & - \\
Pneumocystis carinii & - & - & - & 2 & - \\
Total number & 13 & 8 & 2 & 13 & 2 \\
\hline BC.
\end{tabular}

BC: blood culture; PSB: protected specimen brush; BAL: bronchoalveolar lavage; TBAS: tracheobronchial aspirate.

The diagnostic results of the diverse techniques are listed in table 3.

All patients had been pretreated with antimicrobial regimen prior to diagnostic evaluation. Antimicrobial treatment was modified according to diagnostic results in 18/ 64 cases $(28 \%)$. Most treatment changes were based on findings in BALF (10 cases) and blood cultures (4 cases).

\section{Necropsy results}

Eighteen necropsies revealed an infectious aetiology, with 26 pathogens involved. Noninfectious aetiologies were detected in 13 cases. Nine cases with infectious aetiology were associated with DAD (CMV $(\mathrm{n}=4), P$. carinii $(\mathrm{n}=1)$, $P$. aeruginosa $(\mathrm{n}=1), P$. aeruginosa plus CMV plus Herpes simplex plus Aspergillus spp. $(\mathrm{n}=1)$, Candida spp. and Aspergillus spp. $(\mathrm{n}=1$ each)). The results are given in detail in table 4.

Necropsy results were coincident with results of diagnostic evaluation intra vitam in 6/14 cases (43\%) with infectious aetiologies (true positive results) and 6/10 (60\%) cases with noninfectious aetiologies (true negative results). In the remaining seven cases, no diagnostic results obtained during the lifetime were available.

Eight infectious aetiologies were exclusively established by necropsy, including two cases with bacterial pneumonia ( $P$. aeruginosa and Enterococcus spp.), one with polymicrobial pneumonia (P. aeruginosa, CMV, and Aspergillus spp.), three cases with pneumonia due to CMV and two cases due to Aspergillus spp.

Of 47 patients with BMT and lethal outcome, necropsy was performed in 22. Pulmonary infection was present in 16. The remaining six disclosed DAD in four cases (three associated with haemorrhage), and pulmonary haemorrhage and bronchopneumonia without pathogen in one case each.

\section{Outcome and prognostic analysis}

The overall mortality was 70/89 (79\%), 23/37 (62\%) in nontransplanted patients and 47/52 (90\%) in bone marrow recipients $(\mathrm{p}<0.01)$. The mortality was $39 / 43(91 \%)$ in all-
Table 4. - Aetiological findings at necropsy $(n=31)$

\begin{tabular}{|c|c|c|}
\hline Aetiology & Cases & Pathogens \\
\hline $\begin{array}{l}\text { Infectious } \\
\text { Enterococcus spp. } \\
\text { Pseudomonas aeruginosa } \\
\text { Cytomegalovirus (CMV) } \\
\text { Herpes simplex virus } \\
\text { Candida spp. } \\
\text { Aspergillus spp. } \\
\text { Pneumocystis carinii } \\
\text { Mycobacterium tuberculosis } \\
\text { Polymicrobial } \\
\text { P. aeruginosa + Enterococcus spp. } \\
\text { P. aeruginosa + Candida spp. } \\
\text { P. aeruginosa + CMV + Aspergillus spp. } \\
\text { P. aeruginosa + CMV + Herpes simplex } \\
\text { virus + Aspergillus spp. }\end{array}$ & $\begin{array}{c}18 \\
0 \\
1 \\
6\left(3^{*}\right) \\
0 \\
1 \\
3\left(2^{*}\right) \\
1 \\
1 \\
5 \\
2^{*} \\
1 \\
1 * \\
1\end{array}$ & $\begin{array}{r}26 \\
2 \\
6 \\
8 \\
1 \\
2 \\
5 \\
1 \\
1\end{array}$ \\
\hline $\begin{array}{l}\text { Noninfectious } \\
\text { Diffuse alveolar damage } \\
\text { Pulmonary haemorrhage } \\
\text { Others } \\
\text { Diffuse alveolar damage + haemorrhage } \\
\text { Bronchopneumonia + haemorrhage } \\
\text { Organizing pneumonia } \\
\text { Fat embolism }\end{array}$ & & $\begin{array}{l}6 \\
3 \\
4 \\
1 \\
1 \\
1 \\
1\end{array}$ \\
\hline
\end{tabular}

*: episodes not detected in patients with microbiological evaluation during lifetime.

ogeneic and seven of eight (87\%) in autologous transplant recipients $(\mathrm{p}=\mathrm{NS})$. In patients requiring mechanical ventilation, the mortality was $68 / 76(90 \%)$, as compared to $2 / 13$ $(15 \%)$ in those not requiring it $(\mathrm{p}<0.0001)$.

An infectious aetiology was associated with the highest mortality $(32 / 37,87 \%)$, a noninfectious aetiology with the lowest $(15 / 24,63 \%)(\mathrm{p}<0.05)$. An undetermined aetiology had a mortality rate similar to infectious aetiologies (23 of $28,82 \%)(\mathrm{p}=\mathrm{NS})$. The mortality rates for different pathogens were as follows: CMV pneumonia $100 \%$ (11 of 11 , including three mixed infections); fungal pneumonia $91 \%$ (five of six Candida pneumonia, five of five Aspergillus pneumonia, including three mixed infections); bacterial pneumonia $86 \%$ (17 of 21 , including six mixed bacterial/ opportunistic infections); P. aeruginosa pneumonia $100 \%$ (10 of 10, including eight mixed bacterial/opportunistic infections), and P. carinii pneumonia 50\% (one of two, including one mixed infection).

Of the five BMT recipient survivors, none had any definite infectious aetiology of pulmonary compromise and only two required mechanical ventilation. Of these, one had been transplanted 240 days before and was mechanically ventilated because of cardiogenic oedema; a suspected pneumonia was not proven. The other patient had been transplanted 335 days before and had interstitial pneumonitis. They were ventilated for 9 and 19 days, respectively.

Multivariate analysis of adverse prognostic factors in the whole population including the parameters of mechanical ventilation and presence of BMT revealed that both parameters were independent predictors of death (table 5).

In patients without BMT $(n=37)$, the only parameter significantly associated with death was the requirement of mechanical ventilation. In bone marrow recipients $(n=52)$, the requirement of mechanical ventilation and an interval of $<90$ days from BMT to ICU admission were significantly associated with death. Both parameters remained independent predictors of death in the multivariate analysis (table 5). 
Table 5. - Results of uni- and multivariate analysis of prognostic factors in patients with haematological malignancies and pulmonary complications

\begin{tabular}{|c|c|c|c|}
\hline & $\begin{array}{l}\text { Relative } \\
\text { risk }\end{array}$ & $95 \% \mathrm{CI}$ & p-value \\
\hline \multicolumn{4}{|l|}{ Total population (n=89) } \\
\hline \multicolumn{4}{|l|}{ Univariate analysis } \\
\hline Mechanical ventilation & 8.0 & $4.9-16.1$ & $<0.0001$ \\
\hline BMT & 3.9 & $1.6-9.9$ & $<0.01$ \\
\hline Multivariate analysis & 43.7 & $7.3-260.2$ & $<0.0001$ \\
\hline $\begin{array}{l}\text { Mechanical ventilation } \\
\text { BMT }\end{array}$ & 5.2 & $1.2-22.0$ & $<0.05$ \\
\hline \multicolumn{4}{|l|}{ Patients without BMT $(n=37)$} \\
\hline \multicolumn{4}{|l|}{ Univariate analysis } \\
\hline $\begin{array}{l}\text { Mechanical ventilation } \\
\text { BMT recipients }(n=52)\end{array}$ & 4.2 & $1.9-8.7$ & $<0.001$ \\
\hline \multicolumn{4}{|l|}{ Univariate analysis } \\
\hline Mechanical ventilation & 18.0 & $4.1-78.3$ & $<0.0001$ \\
\hline $\begin{array}{l}\text { Interval from BMT to ICU } \\
\text { admission }<90 \text { days }\end{array}$ & 22.0 & $2.8-172.2$ & $<0.0001$ \\
\hline \multicolumn{4}{|l|}{ Multivariate analysis } \\
\hline Mechanical ventilation & 25.7 & $1.0-642.5$ & $<0.05$ \\
\hline $\begin{array}{l}\text { Interval from BMT to ICU } \\
\text { admission }<90 \text { days }\end{array}$ & 21.2 & $1.4-321.3$ & $<0.05$ \\
\hline
\end{tabular}

95\% CI: p5\% confidence interval; BMT: bone marrow transplantation; ICU: intensive care unit.

\section{Discussion}

The main findings of the present study are as follows: 1) The aetiology was infectious in $42 \%$, noninfectious in $27 \%$ and remained undetermined in $31 \%$ of patients with pulmonary compromise. 2) Blood cultures and cytological examinations of BALF were the diagnostic tools with the highest yield (30\% and $29 \%$ positive results, respectively). However, necropsy results were coincident with results obtained during lifetime only in $43 \%$ of cases with infectious aetiologies and $60 \%$ with noninfectious aetiologies. 3) Patients with haematological malignancies and severe pulmonary complications have a high mortality (79\%). The requirement of mechanical ventilation, BMT as well as an interval $<90$ days from BMT to ICU admission were independent adverse prognostic factors.

The principal cause of pulmonary complications in our population was infection. The most frequent infectious aetiology was bacterial pneumonia, with $P$. aeruginosa representing the leading bacterial pathogen. However, nearly $30 \%$ of bacterial pneumonias and $50 \%$ of episodes with $P$. aeruginosa were mixed bacterial/opportunistic infections. Dunagan et al. [15], in a recent report about bronchoscopic evaluation of pulmonary infiltrates in bone marrow recipients also found bacteria as the most common aetiology but Gram-positive pathogens as the most frequent pathogens. Pneumonia due to CMV was the most frequent single aetiologic agent, and as expected occurred signifi-cantly more frequently in transplanted patients as compar-ed with the nontransplanted group. However, the incidence of infection due to CMV has ultimately changed with the practice of providing CMV-negative screened blood to CMV-seronegative marrow recipients, the prophylactic use of hyperimmune-CMV-Igs, acyclovir or ganciclovir, and pre-emptive therapy $[16,17]$. This is also the reason for a clear recent reduction of admissions in our ICU with this type of pulmonary complication. Thus, fungal pneumonia due to Candida spp. and Aspergillus spp. may emerge as leading pathogens in both transplant recipients and nontransplanted patients [18]. Fungal pneumonia due to Candida spp. and Aspergillus spp. was frequently encountered in our study. The mortality rate of infectious complications was generally high $(87 \%)$. Noninfectious aetiologies were mostly due to pulmonary haemorrhage and heart fail-ure, and the mortality rate in these patients was lower than in patients with infectious aetiologies $(63 \%)$. A considerable number of episodes remained undetermined. The mortality rate in this latter group of patients was comparable with that infectious aetiologies (82\%). This group may include episodes of missed infectious aetiologies, especially bacterial pneumonia, but also idiopathic pneumonia syndrome (IPS) and a variety of inflammatory conditions like DAD, bronchiolitis obliterans with organizing pneumonia (BOOP), bronchiolitis obliterans (BOP), and pulmonary GVHD. The incidence of IPS has been estimated to reach $10-20 \%[19,20]$, and inflammatory conditions as diagnosed by lung histology were found in up to $10 \%$ of episodes [19]. As expected, DAD as a uniform result of infectious and noninfectious lung injury was a rather frequent finding on necropsy, with a comparable incidence in patients with and without infection (50 versus $46 \%$ ).

Bacterial and fungal cultures of respiratory samples retrieved by TBAS, PSB and BALF had a poor diagnostic yield $(6,20$ and $4 \%$, respectively). Conversely, cytological examinations of BALF were diagnostic in $29 \%$ of the cases investigated. These observations can be explained, on the one hand, by extensive antimicrobial pretreatment impeding growth in culture of respiratory secretions and on the other hand, by the fact that the yield of cytological examinations for respiratory pathogens is less affected by antimicrobial pretreatment. Previous studies investigating bronchoscopic techniques in bone marrow recipients found a yield ranging from $42-66 \%$ for bronchoalveolar lavage $[3,21-23]$ and $31-74 \%$ for a combined procedure including transbronchial biopsies $[4,15,19]$. Conflicting results were also reported for the yield of bronchoalveolar lavage in non-transplanted patients with acute leukaemia, with yields ranging from 15-60\% [24, 25]. Differences in the severity of pulmonary compromise, the timing of bronchoscopy, guidance of bronchoscopic sampling by computed tomography (CT)-scan of the chest, prophylaxis and antimicrobial pretreatment regimens and microbiological testing may account for the discrepancies in the reported diagnostic yields. In our study, the concordance of diagnostic evaluation during lifetime and necropsy was poor. A significant amount of infectious agents was missed by diagnostic techniques. Three episodes of CMV pneumonia were exclusively diagnosed at necropsy, and only one of five cases with Aspergillus pneumonia was diagnosed during lifetime in our study. Whereas new diagnostic tools like shell vial cultures, detection of antigenemia, and CMV deoxyribonucleic acid (DNA) in blood leucocytes by polymerase chain reaction (PCR) have recently significantly improved the diagnostic yield of CMV infection [26], the diagnosis of fungal pneumonia, first of all Aspergillus pneumonia, remains particularly troublesome [24, 25, 27, 28]. Recent approaches in the diagnosis of Aspergillus pneumonia using a CT scan of the chest have shown promising results and may obviate the need for an invasive diagnostic confirmation in a considerable amount of cases [29-31]. Nevertheless, the definite diagnosis of viral as well as fungal pneumonia may require histology, which 
carries a considerable risk for complications in these severely disabled and mostly thrombocytopenic patients [4, $15,19]$. In any case, our study suggests that for an initial microbiological diagnostic evaluation of these patients blood cultures (with a yield of $30 \%$ in our study) together with culture and cytology of bronchoalveolar lavage still represent the most adequate techniques. Notwithstanding, antimicrobial treatment will have to rely largely on empirical approaches.

Our study confirms previous reports demonstrating a high mortality rate in patients with haematological malignancies and severe pulmonary complications [5-10]. PETERS et al. [6], in a study including 119 episodes of mechanical ventilation in 116 patients with haematological malignancies, reported an in-hospital mortality of $82 \%$. SCHUSTER and MARION [11] found a mortality of $80 \%$ in 77 cases treated in the ICU. We previously reported a mortality of $80 \%$ in a series of 30 patients treated in our ICU [5]. The present study found a very similar mortality rate $(79 \%)$.

Appropriate initial empirical antimicrobial treatment, especially the early introduction of antifungal treatment, is one crucial issue with regard to the outcome of these patients with pulmonary complications $[2,31]$. The antimicrobial treatment approach was consistent during the study period in that broad spectrum antibiotics with anti-Pseudomonas activity were administered initially and antifungal treatment added after the 4th or 5th day in case of nonresponse to antibacterial treatment. As regards CMV, despite major advances in the prevention of pneumonia, the outcome continues to be unfavourable, despite treatment, once pneumonia has developed. Since growth factors have not been shown to improve the outcome of pulmonary complications, its application in sporadic cases is not regarded as a potential confounder in prognostic analysis. The conditioning regimen in bone marrow recipients did not change during the study period. Previous studies have reported an increased mortality from pneumonia in patients with allogeneic as compared with autologous BMT [32]. In our population, with a limited number of patients with autologous BMT, mortality from pulmonary complications was not different to that with allogeneic BMT.

Mortality was independently associated with the requirement of mechanical ventilation and bone-marrow transplantation. An infectious aetiology was associated with a significantly higher mortality than a noninfectious aetiology, but the mortality from an infectious aetiology was not significantly different as compared to the mortality of complications with an undetermined aetiology.

Bone-marrow recipients with pulmonary compromise requiring ICU admission represent a subgroup with a particularly poor prognosis. It has been shown previously that the requirement of mechanical ventilation is an important predictor of mortality in these patients. Mortality rates in these patients ranged between 73 and 96\% [7-10, 33]. Moreover, survival rates rapidly dropped after discharge and reached 3\% after 6 months $[9,33]$. In the present study, 52 transplanted patients were admitted to the ICU, and $47(90 \%)$ died. The mortality rate was independently associated with mechanical ventilation. The mortality rate was $100 \%$ in bone marrow recipients requiring mechanical ventilation and concomitant infectious aetiologies. Thus, noninfectious pulmonary complications in bone-marrow recipients may offer more favourable therapeutic prospects, even when mechanical ventilation is required.

The interval from BMT to the development of pulmonary complications has also been related to the outcome. FABER-LANGENDOEN et al. [9] found that bone marrow re-cipients with pulmonary complications developing $<90$ days after transplantation and being mechanically ventilated had a significantly worse outcome than those occurring after $\breve{S} 90$ days. This finding could be confirmed in our study. The interval $<90$ days from BMT to ICU ad-mission was a second independent predictor of death in bone marrow recipients. The high mortality in this period is probably mainly due to opportunistic infections in the presence of severe immunodepletion [16].

The present study is limited to a retrospective survey of a $10 \mathrm{yr}$ series of patients treated at a RICU. Nevertheless, despite considerable progress in the prevention of pulmonary complications after 1990, particularly pneumonia due to $\mathrm{CMV}$, these complications continue to impose diagnostic dilemmas and to be associated with a virtually unchanged poor prognosis. Our study includes a diagnostic evaluation based on a considerable number of necropsies. Moreover, it offers perspectives on populations with different prognostic implications which certainly should be assessed in future prospective trials.

In conclusion, patients with haematological malignancies and severe pulmonary complications present with a variety of aetiologies that can only be incompletely assessed by conventional diagnostic techniques. However, the requirement of mechanical ventilation is the main adverse prognostic factor in both transplanted and nontransplanted patients. Bone-marrow recipients, especially those developing pulmonary complications $<90$ days after transplantation and requiring mechanical ventilation represent a subgroup with a dismal prognosis. Since intensive care unit treatment with mechanical ventilation bears only a marginal, if any, potential to improve the outcome in this population, emphasis should be placed on the development of better preventive regimen for infectious pulmonary complications [34].

\section{References}

1. Wardman AG, Milligan DW, Child JA, Delamore IW, Cooke NJ. Pulmonary infiltrates and adult acute leukemia: empirical treatment and survival related to the extent of pulmonary disease. Thorax 1984; 39: 568-571.

2. Maschmeyer G, Link H, Hiddemann W, et al. Pulmonary infiltrations in febrile patients with neutropenia. Cancer 1994; 73: 2296-2304.

3. Cordonnier C, Bernaudin JF, Bierling P. Pulmonary complications occurring after allogeneic bone marrow transplantation. Cancer 1986; 58: 1047-1054.

4. Campbell JH, Blessing N, Burnett AK, Stevenson RD. Investigation and management of pulmonary infiltrates following bone marrow transplantation: an 8 year review. Thorax 1993; 48: 1248-1251.

5. Estopa R, Torres A, Kastanos N, Rives A, Rozman C. Acute respiratory failure in severe hematologic disorders. Crit Care Med 1984; 12: 26-28.

6. Peters SG, Meadows JA, Gracey DR. Outcome of respiratory failure in hematologic malignancy. Chest 1988; 94 : 99-102.

7. Crawford SW, Schwartz DA, Petersen FB, Clark JG. Mechanical ventilation after marrow transplantation. Risk 
factors and clinical outcome. Am Rev Respir Dis 1988; 137: 682-687.

8. Torrecilla C, Cortes JL, Chamorro C, et al. Prognostic assessment of acute complications of patients with bone marrow transplantation requiring intensive therapy. Intensive Care Med 1988; 114: 393-398.

9. Faber-Langendoen K, Caplan AL, McGlave PB. Survival of adult bone marrow transplant patients receiving mechanical ventilation: a case for restricted use. Bone Marrow Transplant 1993; 12: 501-507.

10. Paz H, Crilley P, Weinar M, Brodsky I. Outcome of patients requiring medical ICU admission following bone marrow transplantation. Chest 1993; 104: 527-531.

11. Schuster DP, Marion JM. Precedents for meaningful recovery during treatment in a medical intensive care unit. Am J Med 1983; 75: 402-408.

12. Murray JF, Mattay MA, Luce JM, Flick MR. An expanded definition of the adult respiratory distress syndrome. Am Rev Respir Dis 1988; 138: 720-723.

13. Torres A, Pulg de la Bellacasa J, Xaubet A, et al. Diagnostic value of quantitative cultures of bronchoalveolar lavage and telescoping plugged catheters in mechanically ventilated patients with bacterial pneumonia. Am Rev Respir Dis 1989; 140: 306-310.

14. Kahn FW, Jones JM, England DM. Diagnosis of pulmonary hemorrhage in the immunocompromised host. Am Rev Respir Dis 1987; 136: 155-160.

15. Dunagan DP, Baker AM, Hurd DD, Haponik EF. Bronchoscopic evaluation of pulmonary infiltrates following bone marrow transplantation. Chest 1997; 111: 135-141.

16. Crawford SW. Bone marrow transplantation and related infections. Sem Respir Infect 1993; 8: 183-190.

17. Armitage JO. Bone marrow transplantation. $N$ Engl $J$ Med 1994; 330: 827-828.

18. Pannuti C, Gingrich R, Pfaller M, Chi Kao MA, Wenzel $\mathrm{R}$. Nosocomial pneumonia in patients having bone marrow transplant. Attributable mortality and risk factors. Cancer 1992; 69: 2653-2662.

19. White P, Bonacum JT, Miller CB. Utility of fiberoptic bronchoscopy in bone marrow transplant patients. Bone Marrow Transplant 1997; 20: 681-687.

20. Clark JG, Hansen JA, Hertz MI, et al. Idiopathic pneumonia syndrome after bone marrow transplantation. Am Rev Respir Dis 1993; 147: 1601-1606

21. Cordonnier C, Bernaudin JF, Fleury J. Diagnostic yield of bronchoalveolar lavage in pneumonitis occurring after allogeneic borrow marrow transplantation. Am Rev Respir Dis 1985; 132: 1118-1123.
22. Milburn HJ, Prentice HG, DuBois RM. Role of bronchoalveolar lavage in the evaluation of interstitial pneumonitis in recipients of bone marrow transplantation. Thorax 1987; 42: 766-778.

23. Abu-Farsakh HA, Katz RL, Atkinson N, Champlin RE. Prognostic factors in bronchoalveolar lavage in 77 patients with bone marrow transplants. Acta Cytologica 1995; 39: 1081-1988.

24. Marra R, Pagano L, Pagliari G, et al. The yield of bronchoalveolar lavage in the etiological diagnosis of pneumonia in leukemia and lymphoma patients. Eur J Hematol 1993; 51: 256-258.

25. Saito H, Anaissie EJ, Morice RC, Dekmezian R, Bodey GP. Bronchoalveolar lavage in the diagnosis of pulmonary infiltrates in patients with acute leukemia. Chest 1988; 94: 745-749.

26. Ljungman P, de Bock R, Cordonnier C, et al. Practices for cytomegalovirus diagnosis, prophylaxis and treatment in allogeneic bone marrow transplant recipients: a report from the Working Party for Infectious Diseases of the EBMT. Bone Marrow Transplant 1993; 12: 399-403.

27. Albelda SM, Talbot GH, Gerson SL, Miller WT, Cassileth PA. Role of fiberoptic bronchoscopy in the diagnosis of invasive pulmonary aspergillosis in patients with acute leukemia. Am J Med 1984; 76: 1027-1034.

28. Kahn FW, Jones JM, England DM. The role of bronchoalveolar lavage in the diagnosis of invasive pulmonary aspergillosis. Am J Clin Pathol 1986; 86: 518-523.

29. Kuhlmann JE. The role of chest CT in the evaluation of the febrile bone marrow transplant recipient. Chest 1991; 99: 794-795.

30. Kuhlmann JE, Fishman E, Siegelman S. Invasive pulmonary aspergillosis in acute leukemia: characteristic findings in CT, the CT halo sign, and the role of CT in early diagnosis. Radiology 1985; 157: 611-619.

31. von Eiff M, Zühisdorf M, Roos N, Hesse M, Schulten R, van de Loo J. Pulmonary fungal infections in patients with hematological malignancies - diagnostic approaches. Ann Hematol 1995; 70: 135-141.

32. Gentile G, Micozi A, Girmenia C, et al. Pneumonia in allogeneic and autologous bone marrow recipients. A retrospective study. Chest 1993; 104: 371-375.

33. Crawford SW, Petersen FB: Long-term survival from respiratory failure after marrow transplantation for malignancy. Am Rev Respir Dis 1992; 145: 510-514.

34. Momin F, Chandrasekar PH. Antimicrobial prophylaxis in bone marrow transplantation. Ann Intern Med 1995; 123: 205-215. 